\title{
L'équilibre entre deux rôles \\ dans la recherche sur la fin de vie
}

par Wanda Martin, Meredith Grey, Terry Webber, Linnea Robinson, Nancy Hartt, Moira Cairns et Kelli Stajduhar

\section{Abrégé}

Les études de recherche réalisées auprès de populations recevant des soins palliatifs suscitent presque immanquablement des questions d'ordre éthique et pratique. Certaines infirmières se sentent opportunistes, d'autres se sentent peu disponibles sur le plan affectif tandis que d'autres encore constatent avec désarroi un écart entre les services prodigués et les besoins des participants recevant des soins, mais toutes les infirmières qui participent à la recherche trouvent une façon de répondre à la fois aux besoins des participants et à ceux de l'étude (McIlfatrick, Sullivan et McKenna, 2006). Cette étude illustre certaines des questions d'ordre pratique et éthique qui surviennent lorsque des infirmières de première ligne acceptent de contribuer, à titre d'assistantes de recherche, à des études auprès de populations recevant des soins palliatifs. Plus particulièrement, les auteures présentent leurs expériences personnelles, acquises dans le cadre de leur travail d'assistantes de recherche dans une étude sur la capacité d'adaptation des aidants naturels dans la prestation de soins de fin de vie à des personnes atteintes d'un cancer. Les auteures présentent les difficultés associées à l'exécution de ce double rôle et offrent des recommandations concernant la façon d'aborder - et d'être avec les gens dans le cadre d'un projet de recherche sur la fin de vie et ce, en utilisant un cadre conceptuel fondé sur la théorie du caring de Swanson (1991).

Les soins infirmiers constituent une profession d'une grande étendue qui offre l'occasion d'assumer différents rôles dans toute une gamme de milieux. En 2005, presque $75 \%$ des infirmiers et infirmières du Canada travaillaient en soins directs aux patients, et seulement $0,7 \%$ d'entre eux travaillaient avant tout en recherche (ICIS, 2006). Les infirmières qui se consacrent aux soins directs aux patients possèdent des connaissances particulières et une façon d'être avec les patients et leur famille qui font d'elles d'excellentes ressources pour les chercheurs lors de la collecte de données. Cependant, la participation à des études de recherche oblige ces infirmières à remplir deux rôles : infirmières de première ligne et collaboratrices dans un projet de recherche (Colbourne et Sque, 2004). Il arrive souvent que les infirmières soient préoccupées par des conflits entre leurs obligations envers les participants, d'une part, et envers l'étude, d'autre part : elles doivent « jongler » avec les sensibilités et vulnérabilités des participants tout en tentant de répondre aux exigences de l'étude (Daly et Rosenfeld, 2003). Cette étude illustre certaines des questions d'ordre éthique et pratique qui surviennent lorsque les infirmières adoptent le double rôle de clinicienne et de chercheuse, en particulier auprès de populations en fin de vie. Les auteures discutent de cet exercice de haute voltige et offrent des recommandations concernant la façon d'aborder - et d'être avec les gens dans le cadre d'un projet de recherche sur la fin de vie. Les auteures ont utilisé la théorie du caring de Swanson (1991) comme cadre de discussion.

\section{Contexte}

Les auteures de cet article se sont connues lorsqu'elles ont été embauchées pour travailler dans un projet de recherche à sites multiples subventionné par la Société canadienne du cancer et baptisé «Aidants naturels face à des soins de fin de vie liés au cancer ». Le projet était dirigé par une infirmière chercheuse (K.S.), était coordonné par une infirmière titulaire d'une maîtrise (W.M.) et regroupait plusieurs assistantes de recherche, dont cinq étaient des infirmières qui ont été chargées de réaliser des entrevues semi-structurées approfondies et d'administrer un questionnaire structuré à un échantillon d'aidants naturels dont un proche était en train de mourir du cancer. L'objectif principal de l'étude consistait à déterminer la raison pour laquelle certains aidants naturels semblent mieux s'adapter que d'autres à des situations semblables. Les assistantes de recherche ne contribuaient pas directement à la prestation de soins aux patients mourants, mais œuvraient dans le domaine des soins infirmiers à domicile ou en oncologie. Elles possédaient une expérience et un savoir-faire spécialisés auprès de la population cible. On a recruté les participants à l'étude dans des centres de soins palliatifs, des programmes de soins à domicile et des cliniques de gestion de la douleur et des symptômes dans trois centres urbains de la Colombie-Britannique. On a réalisé des entrevues qualitatives auprès de 28 aidants actuels et de 17 aidants en deuil. Plus de 300 aidants naturels ont participé à la phase quantitative de l'étude. Bon nombre des assistantes de recherche avaient une formation de recherche formelle limitée; afin de les préparer au projet, la chercheuse principale leur a offert deux séances de formation d'une journée complète chacune. La formation couvrait des sujets comme

Wanda Martin, inf, MscInf, Coordonnatrice du projet, Centre on Aging, Université de Victoria, Sedgewick Building, Room A104, 3800 Finnerty Road, Victoria, C.-B. V8P 5C2, Courriel : wmartin@uvic.ca

Meredith Grey, inf, BScInf, Conseillère en amélioration de la qualité - Santé cardiaque et Unité de soins intensifs pour adultes, Quality and Patient Safety, Vancouver Island Health Authority, Victoria, C.-B.

Terry Webber, inf, Assistante de recherche, Université de Victoria, Infirmière clinicienne-ressource, Hospice Palliative Care Consult Team, Surrey Memorial Hospital, Surrey, C.-B.

Linnea Robinson, inf, BScInf, Assistante de recherche, Université de Victoria, Home Health Nurse (LTC), Fraser Health Authority, White Rock, $C .-B$.

Nancy Hartt, inf, BScInf, Assistante de recherche, Université de Victoria, Infirmière en essais cliniques et infirmière en oncologie, BC Cancer Agency - Centre for the Southern Interior Kelowna, C.-B.

Moira Cairns, BA, RSW, Assistante de recherche, Université de Victoria, Victoria, $C .-B$.

Kelli I. Stajduhar, inf, PhD, Professeure adjointe, School of Nursing and Centre on Aging, Université de Victoria, Victoria, C.-B. 
la conception d'une étude, les techniques d'entrevue, la déontologie de recherche et les aspects pragmatiques de la participation à un projet de recherche.

Comme l'étude n'est pas encore tout à fait terminée, les résultats n'en sont toujours pas disponibles. Cependant, lors de l'entrevue des aidants naturels, diverses questions d'ordre pratique et éthique ont fait surface. Ces questions étaient liées au rôle double que les assistantes de recherche assumaient. Les membres de l'équipe de recherche (la chercheuse principale, la coordonnatrice du projet et les assistantes de recherche) étaient d'avis qu'il serait intéressant d'explorer ces doubles rôles et la signification qu'ils avaient pour les assistantes. Bien que la question n'était pas conçue comme une étude de recherche, l'équipe croyait que les leçons tirées de ses expériences collectives pourraient profiter à d'autres infirmières en oncologie et en soins palliatifs qui assumaient également des rôles en recherche. La coordonnatrice du projet a commencé à prendre note des questions qui émergeaient relativement à ces rôles doubles, et quatre des assistantes de recherche lui ont fourni un journal dans lequel elles avaient consigné leurs pensées et émotions par rapport à leur expérience de travail dans le cadre du projet de recherche. Cette information a formé la base de cet article. En vue de mettre de l'ordre dans cette information, les auteures ont utilisé comme cadre de travail la théorie du caring de Swanson (1991), pour décrire les rôles doubles des infirmières chercheuses. Les piliers de cette théorie de portée moyenne sont les suivants : « savoir », « être avec l'autre », « agir pour l'autre », " habiliter l'autre » et «maintenir la conviction ». Cette théorie du caring est tout à fait pertinente pour décrire la pratique infirmière clinique. Cependant, du point de vue de la recherche, la pertinence de la théorie n'est pas aussi évidente, puisque la collecte active de données dans le cadre d'un projet de recherche peut s'avérer peu compatible avec la pratique clinique.

\section{"Savoir"}

Selon Swanson (1991), « savoir » signifie approcher une situation sans présuppositions, en se centrant plutôt sur la personne qui reçoit les soins et en évaluant son expérience afin de tenter de comprendre sa réalité. Savoir du point de vue des soins infirmiers était presque instinctif pour certaines des infirmières de ce projet, qui travaillent depuis un grand nombre d'années dans le domaine des soins directs aux patients. Mais savoir du point de vue de la recherche était pour elles une expérience moins familière.

La démarche adoptée dans l'étude sur la capacité d'adaptation des aidants naturels devait être axée sur les participants, non seulement pour comprendre leurs réalités, le but et la raison d'être de l'étude, mais également afin de les respecter comme personnes. Bien que cette méthode correspondait au savoir des infirmières, à certaines occasions les assistantes de recherche se retrouvaient dans des situations dans lesquelles elles devaient choisir entre leur rôle d'infirmière et leur rôle de chercheuse. Par exemple, elles éprouvaient souvent un conflit entre leur rôle de soignantes et prestataires de soins et la sensation qu'elles avaient d'être opportunistes. Elles avaient parfois l'impression qu'elles exploitaient la famille ou la personne mourante pour remplir un programme de recherche. Ces sentiments avaient tendance à faire surface le plus souvent dans le cadre des entrevues quantitatives, qui étaient structurées et laissaient moins de temps aux participants de réellement explorer leurs expériences et de les exprimer pleinement.

L'administration des entrevues qualitatives approfondies était plus satisfaisante pour certaines des assistantes de recherche, puisque ces entrevues donnaient l'occasion d'explorer les expériences et les réalités des participants et la façon dont ils composaient avec les exigences de la fourniture des soins. Cette approche permettait aux participants de relater leurs histoires de façon plus complète. Il était évident dans de nombreuses entrevues que le fait d'être interviewé donnait aux participants la chance de se faire entendre et de mieux cerner leur propre capacité de composer avec leur expérience et d'en tirer des leçons. Comme nous l'avons déjà mentionné, les entrevues quantitatives avaient tendance à être moins satisfaisantes que les entrevues qualitatives, puisqu'elles se prêtent moins que ces dernières aux échanges conversationnels. Bien que l'entrevue quantitative ne signifie pas automatiquement une absence de compassion, les auteures ont constaté qu'elle peut parfois restreindre la qualité des échanges avec les participants.

Afin d'éviter les sentiments d'opportunisme, il est essentiel que les assistantes de recherche reconnaissent le rôle bénéfique de leur présence réceptive pour le patient, peu importe le style de l'entrevue (qualitatif ou quantitatif). Lorsqu'on aborde la recherche à partir d'une théorie du caring, les assistantes de recherche sont moins susceptibles d'exploiter la relation avec le patient au profit du programme de recherche (Head et Ritchie, 2004). Les assistantes obtenaient le consentement éclairé des patients de participer à l'étude et respectaient leurs décisions concernant le niveau de participation auquel ils étaient disposés à s'engager. Le maintien de ces normes de recherche aidait à la fois les participants et les assistantes de recherche à prévenir les sentiments négatifs associés à l'opportunisme.

Cependant, tout projet de recherche sur la fin de vie présente forcément une foule de questions d'ordre éthique. Les chercheuses et les membres de leur équipe doivent se souvenir que de nombreux participants sont altruistes et éprouvent une grande satisfaction à l'idée de contribuer à des travaux de recherche susceptibles d'aider autrui (Head et Ritchie, 2004). Par conséquent, quelle qu'elle soit, la contribution des participants peut s'avérer très utile au processus. Les entrevues qualitatives permettent d'avoir des conversations plus en profondeur, tandis que les entrevues quantitatives sont fondées sur des questions directes, mais les deux ont un rôle à jouer. La connaissance des normes déontologiques de la recherche et le respect des décisions des participants concernant leur degré de participation aideront les infirmières chercheuses et assistantes de recherche à trouver un équilibre entre leurs deux rôles.

\section{"Être avec l'autre"}

La deuxième composante du processus de caring, « être avec l'autre », signifie faire preuve d'ouverture affective, faire comprendre à la personne que son expérience est importante et partager des émotions de façon non imposante (Swanson, 1991). Cette capacité de faire preuve d'empathie est une caractéristique importante en soins infirmiers (Kunyk et Olson, 2001). Auprès de la personne, la simple présence physique ne suffit pas. Les assistantes de recherche signalaient leur besoin de comprendre les réalités des participants du point de vue de la recherche tout en étant émotionnellement présentes. Elles voulaient trouver un équilibre entre les besoins scientifiques analytiques du projet et la nécessité de faire preuve d'empathie dans leur rôle infirmier.

Le titre « infirmière » peut avoir une forte influence sur le processus de recherche. L'identité culturelle des infirmières en tant que praticiennes empathiques habituées à aborder dans leur travail la vie privée des particuliers favorise l'ouverture et donne aux gens la liberté de discuter de tabous sociaux (Leslie et McAllister, 2002). Cette volonté de la part des participants à participer à une étude et à se confier à des assistantes de recherche ouvre des espaces qui pourraient demeurer inaccessibles à des non-infirmières. Cependant, cet avantage n'est pas sans dangers, et les assistantes doivent procéder avec prudence.

Certains participants peuvent ressentir une certaine redevance ou dépendance envers l'infirmière et ainsi accepter de participer à l'étude sans réellement le vouloir (Head et Ritchie, 2004). Le personnel de 
recherche doit posséder une formation et une éducation adéquates lui permettant de reconnaître et d'éviter de telles situations. Il est important à la fois pour les chercheuses et pour les participants de bien définir les rôles afin d'assurer que le consentement est volontaire (Clarke et Johnson, 2003).

Il importe également d'avoir conscience du pouvoir par défaut du rôle infirmier. Face aux patients, les infirmières font office d'enseignantes et de conseillères, et leur rôle habituel est axé sur l'intervention et non sur la collecte de données dans le cadre d'un projet de recherche. L'identité culturelle des infirmières est projetée par autrui, mais elle est également intériorisée par l'infirmière chercheuse (MacIntosh, 2003).

L'ouverture émotionnelle peut mener à un biais de subjectivité pouvant nuire aux résultats de l'entrevue si l'infirmière chercheuse ou l'assistante de recherche perd de vue les objectifs du projet (Field, 1991). En tant que chercheuses, nous devons cerner nos valeurs et croyances, reconnaître notre subjectivité et poursuivre notre autoévaluation tout au long de la période de collecte de données et du processus d'analyse (Polit et Hungler, 1999). Cependant, il existe toujours une tension entre l'utilisation de la recherche à des fins thérapeutiques et son utilisation à des fins de collecte de données. « Être avec l'autre » c.-à-d. le partage des sentiments, et cette utilisation de la communication thérapeutique peut permettre d'alléger la souffrance du participant (Leslie et McAllister, 2002). Une des assistantes de recherche a décrit ainsi son expérience d' " être avec » le participant pendant l'entrevue :

Une des aidantes naturelles était extrêmement perturbée lorsque je lui ai rendu visite. La condition de son mari se détériorait rapidement depuis qu'il avait fait une chute, et il n'était plus capable de se déplacer seul. Il était assis dans la salle d'à côté et appelait fréquemment son épouse. Cette dernière semblait avoir des difficultés à se concentrer sur l'entrevue, en partie à cause des appels de son conjoint et en partie en raison de ses propres préoccupations par rapport à sa conviction qu'elle allait bientôt le perdre. Je lui ai signalé qu'elle pouvait cesser l'entrevue en tout temps, mais elle a insisté que, bien qu'elle n'était pas certaine que son récit me serait utile, cela lui faisait du bien de le partager avec moi. Elle m'a confié : "Mon mari et moi sommes si différents à des moments comme celuici... lorsqu'il est stressé, il se renferme, mais moi, j'ai besoin de parler! »J'étais stupéfaite parce que malgré les moments turbulents qu'elle traversait, elle continuait d'avoir besoin de partager son expérience ouvertement et verbalement.

\section{"Agir pour l'autre" et "habiliter l'autre"}

La troisième et la quatrième composantes de la théorie du caring de Swanson (1991) sont la manifestation physique de la prestation de soins : « Agir pour l'autre » et « habiliter l'autre ». « Agir pour l'autre » implique faire pour une autre personne ce qu'elle ferait pour elle-même si elle le pouvait. Cela comprend le réconfort, l'anticipation, la protection et l'intervention compétente, et il s'agit possiblement des aspects les plus facilement reconnaissables des soins infirmiers (Swanson, 1991). « Habiliter l'autre » signifie aider autrui à traverser des périodes de transition de vie et des événements non familiers en utilisant des connaissances spécialisées. Cela implique l'offre d'information et la prestation d'un soutien affectif tout en validant les sentiments de l'autre (Swanson). Bien que « agir pour l'autre » et « habiliter l'autre » constituent deux catégories distinctes de la théorie de Swanson, nous les regroupons dans ce travail parce nous sommes d'avis que, dans le contexte de ce projet de recherche, les assistantes donnent aux aidants naturels les moyens de traverser une phase de transition de vie au cours de laquelle ils perdent un membre de leur famille.

Souvent, en raison de la tendance qu'ont les infirmières à prodiguer activement des soins, celles qui travaillent dans le domaine de la recherche se sentent partagées entre le rôle familier de l'infirmière attentionnée, empathique et proactive et le besoin de recueillir des données non biaisées et exactes (Asselin, 2003). Cette tension est mise en relief lorsque les infirmières engagées dans la recherche constatent que le participant a un besoin réel qui nécessite une attention, mais qu'elles sont limitées par leur rôle de chercheuses.

Cette situation est très commune, et il est donc extrêmement important que les infirmières qui font de la recherche pour la première fois soient prêtes à faire face à cette tension. Lorsqu'elles ressentent une confusion des rôles, les assistantes de recherche ont avantage à tenir un journal de leurs pensées, sentiments et réactions face aux observations et entrevues (Asselin, 2003). Il est crucial qu'elles aient un solide réseau de soutien. L'éducation de groupe sur la résolution des conflits d'ordre éthique et les techniques de négociation peuvent aider les infirmières assistantes de recherche à composer avec d'éventuelles tensions dans leurs rôles et ce, dès leur survenue. Ces séances peuvent comprendre des exercices de verbalisation et des discussions entre pairs, qui donnent aux infirmières l'occasion de demander conseil et de résoudre les conflits de rôles (Beale et Wilkes, 2001). Finalement, les équipes de recherche fonctionnent particulièrement bien lorsqu'elles sont dirigées par une personne d'expérience qui connaît bien ce besoin émotionnel de prodiguer des soins de façon active et qui peut offrir une orientation et un soutien pertinents (Mitchell et Jones, 2004). Ce leadership est essentiel au soutien des assistantes novices qui sont également des infirmières.

Lorsque les assistantes de recherche éprouvent la troublante sensation qu'un participant ne reçoit pas les soins qu'il devrait recevoir, elles ont trois options : respecter la confidentialité et garder les préoccupations pour soi, rompre la confidentialité et discuter de la situation avec une collègue en vue de répondre aux besoins du participant ou recommander au participant qu'il communique avec son prestataire de soins afin d'aborder la question (Casarett et Karlawish, 2000; Field, 1991). De ces trois options, la troisième est la seule qui réponde à la fois aux besoins du participant et à ceux du projet de recherche. Cependant, si les infirmières engagées dans la recherche comptent prodiguer des soins, elles devraient attendre la fin de la période de collecte de données, si cela est possible sur le plan de l'éthique. À part les situations qui nécessitent une intervention immédiate, les assistantes de recherche devraient éviter la tentation d'intervenir durant l'entrevue et plutôt attendre la fin de l'entrevue pour répondre aux questions ou aiguiller le participant (Colbourne et Sque, 2004). Évidemment, le jugement personnel déterminera le meilleur intérêt du participant, et il se peut que l'on doive mettre fin à l'entrevue pour remplir les exigences déontologiques au sein du projet de recherche.

La prestation de soins au participant comporte une composante émotionnelle, et les assistantes de recherche étaient d'avis qu'il est important de valider immédiatement les sentiments du participant. Ces auteures soutiennent que si l'on ignore une partie des besoins affectifs du participant, il risque de se sentir isolé. Il pourrait également avoir la sensation d'avoir partagé une expérience très intime sans que l'on reconnaisse ses sentiments, ce qui peut faire obstacle au dialogue ouvert. Inversement, si l'assistante de recherche reconnaît les sentiments d'une façon qui accorde trop d'importance à la situation, il se peut que le participant se concentre sur des détails positifs et biaise ainsi la recherche. Il s'agit d'un équilibre délicat, qui nécessite un jugement critique et intuitif pour déterminer si un soutien émotionnel suffisant est offert au moment opportun. Un besoin d'information peut attendre jusqu'à la fin de l'entrevue, mais les besoins affectifs nécessitent souvent une attention immédiate. La dimension « agir pour l'autre » s'avère souvent être un aspect complexe du travail de recherche, puisque les infirmières doivent trouver un équilibre entre les besoins du participant et ceux de l'étude. 


\section{"Maintenir la conviction"}

La dernière catégorie de la théorie de Swanson (1991) concerne le maintien de la conviction. « Maintenir la conviction » consiste à avoir une attitude caractérisée par l'espoir, à offrir un optimisme réaliste tout en aidant l'autre à trouver, maintenir ou retrouver un sens à son expérience (Swanson, 1991).

Au cours de cette étude, certaines des assistantes de recherche ont indiqué qu'elles ressentaient un conflit entre, d'une part, leur rôle de facilitatrices dans une transition de vie mettant en jeu le mourir d'un membre de la famille et, d'autre part, la réaction de certains participants au titre de l'étude («Aidants naturels face à des soins de fin de vie liés au cancer »). Ce titre figurait sur chacun des documents envoyés aux participants. L'équipe de recherche a constaté une fois de plus que le terme « fin de vie » peut constituer une lourde charge émotionnelle pour les personnes qui se trouvent dans une telle situation (Addington-Hall, 2002). Les aidants naturels qui participaient à l'étude signalaient un sentiment de perte d'espoir lorsqu'ils lisaient ces mots. Bien que l'on ait recruté tous les participants par le biais de centres de soins palliatifs, de programmes de soins palliatifs ou de cliniques de gestion de la douleur et des symptômes et qu'ils étaient conscients de la gravité de la maladie du membre de leur famille, le fait de voir ces mots sur le papier suscitait chez certaines personnes de fortes réactions. À titre d'infirmières, les assistantes de recherche ont donc suggéré de retirer le terme « fin de vie » des documents attenants à l'étude afin d'aider les aidants à garder l'espoir. En agissant ainsi à titre de porte-parole, les infirmières intervenaient au nom des participants et en favorisait l'autonomisation. L'étude a bénéficié du fait que les assistantes de recherche étaient également des infirmières, puisque ces dernières saisissaient l'importance de maintenir la conviction au sein de cette population. Lorsque nous avons modifié les documents liés à l'étude, le taux de participation a augmenté de $37,5 \%$.

\section{Références}

Addington-Hall, J. (2002). Research sensitivities to palliative care patients. European Journal of Cancer Care, 11, 220-224.

Asselin, M.E. (2003). Insider research. Issues to consider when doing qualitative research in your own setting. Journal for Nurses in Staff Development, 19(2), 99-103.

Beale, B., \& Wilkes, L. (2001). Nurse researcher: Always a researcher, sometimes a nurse. Collegian, 8(4), 33-39.

Casarett, D.J., \& Karlawish, J.H.T. (2000). Are special ethical guidelines needed for palliative care research? Journal of Pain and Symptom Management, 20(2), 130-139.

Clarke, J.M., \& Johnson, B. (2003). Collecting sensitive data: The impact on researchers. Qualitative Health Research,13(3), 421434.

Colbourne, L., \& Sque, M. (2004). Split personalities: Role conflict between the nurse and the nurse researcher. Journal of Research in Nursing, 9(4), 297-304

Daly, B.J., \& Rosenfeld, K. (2003). Maximizing benefits and minimizing risks in health services research near the end of life. Journal of Pain and Symptom Management, 25(4), S33-S42.

Field, P.A. (1991). Doing fieldwork in your own culture. In J.M. Morse (Ed.), Qualitative Nursing Research: A Contemporary Dialogue (Revised). California: Sage Publications.

Head, B., \& Ritchie, C. (2004). Researching end-of-life care: Challenges, strategies and opportunities. Home Healthcare Nurse, 22(1), 37-44.

\section{Implications}

Des questions d'ordre éthique et pratique surviennent immanquablement dans le cadre de la plupart des études de recherche auprès de populations recevant des soins palliatifs (Addington-Hall, 2002). Les expériences et leçons tirées de ce projet démontrent qu'il est possible de trouver un équilibre entre le rôle infirmier et le rôle d'assistante de recherche. Grâce au leadership d'une infirmière chercheuse expérimentée, à une formation adéquate et à la possibilité de faire le point sur la situation dans le cadre du travail auprès de populations sensibles et vulnérables, les infirmières peuvent réellement contribuer à améliorer les processus de recherche en s'assurant qu'on respecte les normes déontologiques non seulement dans la conception de l'étude, mais également dans le traitement des participants au projet. Tenter d'occulter le côté infirmière des assistantes peut être contraire à la philosophie des soins infirmiers (Colbourne et Sque, 2004) et diminuer le sentiment de satisfaction découlant de la participation à la recherche. Il est possible de trouver un équilibre entre ces deux rôles. La reconnaissance de nos biais par la consignation de nos pensées, d'une part, et les autoévaluations continuelles, d'autre part, sont des outils dont les infirmières peuvent bénéficier tant dans la pratique clinique que dans la recherche. Il peut être bon de demander aux assistantes de recherche qui travaillent en clinique de tenir un journal axé spécifiquement sur l'équilibre entre les deux rôles de chercheuses et d'infirmières. Il se peut que la meilleure façon de résoudre les enjeux consiste à reconnaître, à mesure qu'elles font surface, les demandes conflictuelles propres à chaque rôle (Colbourne et Sque). Les assistantes de recherche qui travaillent dans des domaines particulièrement délicats comme l'oncologie et les soins palliatifs peuvent également bénéficier d'une préparation et d'une formation spécialisées et d'une supervision et d'un soutien continus. Elles seront ainsi mieux préparées à aborder et résoudre les questions difficiles à mesure qu'elles surgissent (Johnson et Clarke, 2003). La recherche future axée sur la manière d'optimiser un équilibre entre ces deux rôles contribuera à réellement tirer profit des compétences des infirmières cliniciennes qui s'engagent dans de la recherche infirmière.

Institut canadien d'information sur la santé. (2006). Tendances de la main-d'œuvre des infirmières et infirmiers autorisés au Canada, 2005. Récupéré le 5 mars 2007 à l'adresse : http://secure.cihi.ca/cihiweb/dispPage.jsp?cw_page=PG_590_F\& cw_topic=590\&cw_rel=AR_20_F

Johnson, B., \& Clarke, J.M. (2003). Collecting sensitive data: The impact on researchers. Qualitative Health Research, 13(3), 421434.

Kunyk D., \& Olson, J.K. (2001). Clarification of conceptualizations of empathy. Journal of Advanced Nursing, 35(3), 317-325.

Leslie, H., \& McAllister, M. (2002). The benefits of being a nurse in critical social research practice. Qualitative Health Research, 12(5), 700-712.

MacIntosh, J. (2003). Reworking professional nursing identity. Western Journal of Nursing Research, 2(6), 725-741.

McIlfatrick, S., Sullivan, K., \& McKenna, H. (2006). Exploring the ethical issues of the research interview in the cancer context. European Journal of Oncology Nursing, 10, 39-47.

Mitchell, T., \& Jones, S. (2004). Leading and co-ordinating a multinurse researcher project. Nurse Researcher, 12(2), 42-55.

Polit, D.F., \& Hungler, B.P. (1999). Nursing research principles and methods (6th ed.). Philadelphia: Lippincott Williams \& Wilkins.

Swanson, K.M. (1991). Empirical development of a middle range theory of caring. Nursing Research, 40(3), 161-6. 